\title{
The Hyades stream: an evaporated cluster or an intrusion from the inner disk?
}

\author{
B. Famaey ${ }^{1}$, F. Pont ${ }^{2}$, X. Luri ${ }^{3}$, S. Udry ${ }^{2}$, M. Mayor ${ }^{2}$, and A. Jorissen ${ }^{1}$ \\ 1 Institut d'Astronomie et d'Astrophysique, Université Libre de Bruxelles, CP 226, Boulevard du Triomphe, 1050 Bruxelles, Belgium \\ e-mail: bfamaey@astro.ulb.ac.be \\ 2 Observatoire de Genève, Chemin des Maillettes 51, 1290 Sauverny, Switzerland \\ 3 Departament d'Astronomia i Meteorologia, Universitat de Barcelona, Avda. Diagonal 647, 08028 Barcelona, Spain
}

Received 26 May 2006 / Accepted 25 September 2006

ABSTRACT

\begin{abstract}
The nature of the Hyades stream, or Hyades moving group, is a long-standing question of Galactic Astronomy. While it has become widely recognized that the Hercules stream, an unbound group of stars lagging behind galactic rotation and moving outward in the galactic disk, is associated with the outer Lindblad resonance of the rotating galactic bar, there is still some debate about the nature of the more prominent low-velocity stream sharing the kinematics of the Hyades open cluster. Is this stream caused by additional nonaxisymmetric perturbations of the galactic potential, such as transient or quasi-stationary spiral waves, or by the on-going evaporation of the Hyades cluster? Here, a simple observational test has been designed to determine whether the Hyades stream is primarily composed of coeval stars originating from the Hyades cluster, or of field stars. Using the Geneva-Copenhagen survey of $F$ and $\mathrm{G}$ dwarfs, we compare the mass distribution and metallicity of the stream to those of field disk stars. If the Hyades stream is composed of stars trapped at resonance, its mass distribution should obey the present-day mass function (PDMF) of the disk, and its metallicity should reflect its origin in the inner regions of the Galaxy. On the other hand, if it is an evaporated cluster, we expect a different mass distribution, depending on the inital mass function (IMF) of the cluster, and on the proportion of evaporated stars as a function of mass. We find that extreme conditions have to be adopted for the selective evaporation and IMF of the cluster to make the observed mass distribution of the stream only roughly consistent (at a one-sigma level) with the coeval evaporated cluster scenario. The observed mass distribution is in much better agreement with the PDMF of the field. The peculiar metallicity of the stream is inconsistent with that of a field population from the solar neighbourhood trapped in the primordial cluster during its formation process and subsequently evaporated. These observations thus favour a resonant origin for the Hyades stream.
\end{abstract}

Key words. Galaxy: kinematics and dynamics - Galaxy: evolution - Galaxy: open clusters and associations: individual: Hyades Galaxy: disk - Galaxy: solar neighbourhood - stars: kinematics

\section{Introduction}

It has long been known that a spatially unbound group of stars in the solar neighbourhood shares the same kinematics as the Hyades open cluster (Hertzsprung 1909; Strömberg 1922; Eggen 1958; Perryman et al. 1998). Assuming that it is a vestige of an initially more massive cluster which partly evaporated with time, Eggen named this kinematically cold group the Hyades supercluster. More generally, it is called the Hyades stream, or Hyades moving group.

During the last fifteen years, Eggen's hypothesis that kinematic groups of this type are in fact cluster remnants has been largely debated, because they may also be generated by a number of global dynamical mechanisms. A rotating bar at the centre of the Milky Way may cause the velocity distribution in the vicinity of the outer Lindblad resonance to become bimodal, due to the coexistence of orbits elongated along and perpendicular to the bar's major axis. Today, this mechanism is thought to account for the Hercules stream (Dehnen 2000), a group of stars lagging behind the galactic rotation and moving outward in the disk. Moreover, the perturbation that the triaxial bar induces on a flat axisymmetric disk does produce some chaos: Fux (2001) showed that when the bar is taken into account, the chaotic regions, decoupled from the regular regions, are more heavily crowded in the region of the Hercules stream in velocity space. On the other hand, other streams, including the Hyades, could still be linked with the spirality of the Galaxy, because any perturbation of the axisymmetric potential is likely to buffet the stars.

Eggen's scenario and the dynamical perturbations are, of course, not mutually incompatible phenomena. Clusters are known to evaporate over time, and there must be some intermediate state when a group of stars that are no longer spatially identifiable still share similar velocities. Indeed, disk stars (most of which move on quasi-circular epicyclic orbits) that formed at the same place and time, and that stayed in the same region of the Galaxy after a few galactic rotations must necessarily have the same period of revolution around the Galactic center, and thus the same guiding-center which in turn implies the same tangential velocity (Woolley 1961). On the other hand, our Galaxy is known to have large spiral perturbations that are likely to have a kinematic effect on the velocity of stars. In the past, the importance of stirring by spiral structure was underestimated because it was thought that spirals heated the disk strongly, and because the amount of heating is observationally constrained. However, Sellwood \& Binney (2002) showed that the dominant effect of spirals is to stir without heating. Thus, the Hyades stream may be an outward-moving stream of stars on horseshoe orbits that cross the corotation of the spiral pattern. The kinematics of $\mathrm{K}$ and $\mathrm{M}$ giant stars in the solar neighbourhood seems to support 


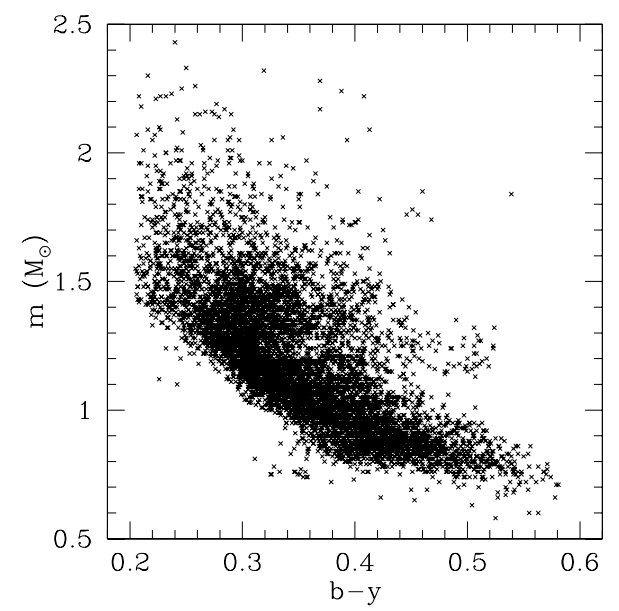

Fig. 1. The distribution of stars from the Geneva-Copenhagen survey in the (b-y-mass) plane.

this scenario, and suggests that the Hyades stream is composed of metal-rich stars with a wide range of ages (Famaey et al. 2005; see also Chereul \& Grenon 2001). However, metallicities were available for too few giants, and individual age estimates were not precise enough to reach a decisive conclusion. This led to the present analysis, where we examine whether properties of $\mathrm{F}$ and $\mathrm{G}$ dwarfs from the Geneva-Copenhagen survey (Nordström et al. 2004) are in better agreement with this dynamical scenario, with Eggen's scenario, or with a mix of both.

\section{The sample}

The Geneva-Copenhagen catalogue of $F$ and $G$ dwarfs (Nordström et al. 2004) is the result of a decade-long campaign of Strömgren photometric and Coravel spectroscopic measurements for more than 16000 late-type dwarfs in the solar neighbourhood. It gives positions, parallaxes, proper motions, masses and metallicities for a sample of dwarfs complete to 40-70 pc depending on spectral type. Metallicities are determined from Strömgren photometry according to a tailored calibration described in Nordström et al. (2004; their Sect. 4.3). Masses are determined by comparing the observed position in the Hertzsprung-Russell diagram (effective temperatures and absolute magnitudes were derived from Strömgren photometry and Hipparcos parallaxes) with stellar-evolution tracks from Girardi et al. (2000). Masses are displayed as a function of the $b-y$ color index in Fig. 1, which shows that the Geneva-Copenhagen survey is only complete in the mass range $[0.8,1.5] M_{\odot}$.

The survey contains 122 stars flagged as definite or possible members of the Hyades cluster by Perryman et al. (1998) and de Bruijne et al. (2001). To avoid contamination from the Hyades cluster in our subsequent analysis of the Hyades stream we exclude those 122 stars from the sample. After also excluding the binaries to avoid the distorting effect of binarity on the photometry, the total number of (single) stars in the present sample is 8084 .

\section{The velocity field}

Figure 2 presents the distribution of all stars from the survey in the $U V$-plane ( $U$ is the velocity towards the galactic center, $V$ the velocity in the direction of Galactic rotation, both with respect to the Sun). To compute $U$ and $V$, distances are adopted from the Geneva-Copenhagen Catalogue. These distances are

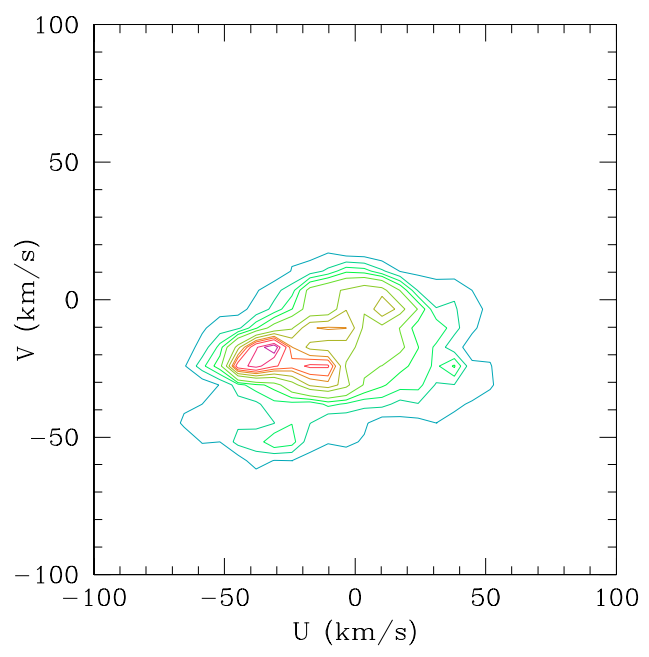

Fig. 2. Isocontours for the whole survey in the $U V$-plane: the contours correspond respectively to $0.5,0.8,1.2,1.5,1.9,2.6,3.1,3.5,3.8,4.2$, $4.7,5$. stars $/\left(\mathrm{km} \mathrm{s}^{-1}\right)^{2}$. The Hyades stream, at $U \simeq-37 \mathrm{~km} \mathrm{~s}^{-1}$ and $V \simeq$ $-17 \mathrm{~km} \mathrm{~s}^{-1}$, is the most prominent feature.

derived from Hipparcos parallaxes if their relative errors are better than $13 \%$, and from photometric distances (which are uncertain by $13 \%$ ) otherwise.

The small-scale structure in the $U V$-plane appears clearly in Fig. 2: the main overdensities are the well-known Hercules stream $\left(U \simeq-30 \mathrm{~km} \mathrm{~s}^{-1}, V \simeq-50 \mathrm{~km} \mathrm{~s}^{-1}\right)$, the Sirius-UMa stream $\left(U \simeq 10 \mathrm{~km} \mathrm{~s}^{-1}, V \simeq-5 \mathrm{~km} \mathrm{~s}^{-1}\right)$, the Castor group $\left(U \simeq-10 \mathrm{~km} \mathrm{~s}^{-1}, V \simeq-10 \mathrm{~km} \mathrm{~s}^{-1}\right)$, the Pleiades stream $\left(U \simeq-15 \mathrm{~km} \mathrm{~s}^{-1}, V=-25 \mathrm{~km} \mathrm{~s}^{-1}\right)$, and, the most prominent, the Hyades stream $\left(U \simeq-37 \mathrm{~km} \mathrm{~s}^{-1}, V \simeq-17 \mathrm{~km} \mathrm{~s}^{-1}\right)$. Those well-known overdensities (see Dehnen 1998; Chereul et al. 1999; Montes et al. 2001; Famaey et al. 2005) are either due to the evaporation of star clusters, or to non-axisymmetric perturbations by spiral arms and the galactic bar (e.g. the Hercules stream). Their prominence close to the center of the $U V$-plane is not compatible with the hypothesis that they are the result of merger events. Substantial amounts of such satellite debris have been identified in the Geneva-Copenhagen survey (Helmi et al. 2006), but at higher velocities and with lower metallicities.

\section{The Hyades stream}

We shall now focus our attention on the prominent Hyades stream, which was suggested by Famaey et al. (2005) to be a dynamical stream coming from the inner Galaxy because of a spiral perturbation, although firm proof was lacking because metallicities were available for only very few stars and individual age estimates were not precise. The main question to be answered here is thus whether this kinematic group is really a dynamical stream, or rather a coeval evaporated cluster.

An evaporated cluster would have a cluster-like homogeneity of age and metallicity, while a dynamical stream would have the age and metallicity composition of a random disk population (its metallicity could however reflect that of the galactocentric radius from which it originates as a result of the dynamical perturbation).

However, even with the high-accuracy data of the GenevaCopenhagen survey, the precision on the metallicity and age is not high enough for one of the scenarios to stand out immediately. Indeed, the ages determined in Nordström et al. (2004) from the position in the HR diagram cannot reliably be used 
(Pont \& Eyer 2004): the uncertainties and systematic biases on the age determination are strongly correlated with the mass, and a group of stars with different masses and homogeneous age cannot be detected as such from the computed ages (see Fig. 25 of Nordström et al. 2004).

Nevertheless, the two scenarios can be distinguished by a more subtle method than the simple analysis of age and metallicity distributions. If the Hyades stream is caused by a nonaxisymmetric dynamical perturbation of the potential, we expect a mass distribution similar to the field stars, obeying the present day mass function (PDMF). On the other hand, if the Hyades stream is an evaporated cluster, composed of coeval stars (600 Myr old), we expect a different mass distribution, depending on the inital mass function (IMF) of the cluster, and on the proportion of escaping stars as a function of mass (see e.g. Terlevich 1987).

\subsection{Predictions in the purely coeval case}

The mass distribution thus offers us a good test of coevality for the Hyades stream. Let us assume that the stream is coeval, entirely generated by the on-going evaporation of the primordial Hyades cluster. We consider two groups of stars, chosen to be as far apart as possible without suffering from selection biases and incompleteness (as illustrated by Fig. 1):

- Group 1: stars with $1.3 M_{\odot} \leq m \leq 1.5 M_{\odot}$. The upper limit is below the Hyades cluster turnoff mass $\left(2.2 M_{\odot}\right)$, so that no giant star from the assumed coeval stream is present in the group. To avoid contamination by the small number of field giants present in the Geneva-Copenhagen survey, we further restrict this group to $b-y<0.42$ (this supplementary condition does not alter the selection for main-sequence stars, see Fig. 1).

- Group 2: stars with $0.8 M_{\odot} \leq m \leq 1 M_{\odot}$.

Then, in this coeval scenario, the proportion of stars belonging to the Hyades stream with respect to field stars of the disk, $N_{\text {hya }} / N_{\text {field }}$, should decrease from the value $p_{1}$ in Group 1 to the value $p_{2}$ in Group 2, according to the variation of the ratio of the mass function of the young evaporated Hyades cluster to the PDMF of field stars. The estimation of the ratio $p_{1} / p_{2}$ under this coeval hypothesis nevertheless depends on the assumptions made on the efficiency of selective evaporation, namely on the proportion of evaporated stars from the cluster in the mass range corresponding to Groups 1 and 2. It is well established that low-mass stars are preferentially depleted from star clusters from the effect of mass segregation (see e.g. Baumgardt \& Makino 2003), leading to a flattening of the observed cluster mass function, or even turning an initially increasing mass function into one that decreases towards low-mass stars (see e.g. Reid \& Hawley 1999; Dobbie et al. 2002). However, it is not obvious that, for a $600 \mathrm{Myr}$ open cluster, a significant preferential depletion should be expected for stars in the range $[0.8,1] M_{\odot}$ as compared to stars in the range $[1.3,1.5] M_{\odot}$.

\subsubsection{Without selective evaporation}

Let us first assume that the relative depletion of stars from the primordial Hyades cluster is the same for the two mass groups under consideration. The values $p_{1}$ and $p_{2}$ are then entirely determined by the ratio of the Hyades cluster IMF to the field PDMF, integrated over the appropriate mass intervals.

Moreover, since by construction Group 1 is restricted to dwarf stars, it is also necessary to correct the PDMF for the absence of field giants when estimating $p_{1}$ : the ratio of the main sequence lifetime over total lifetime is $80 \%$ for a $1.5 M_{\odot}$ star with $Z=0.008$ (Lejeune \& Schaerer 2001, assuming that the evolution posterior to the Helium flash represents $10 \%$ of the MS lifetime), implying a correction of the PDMF by a factor 0.8 in Eq. (1) below. Such a correction is unnecessary for Group 2, dominated by low-mass stars that can leave the main-sequence only when they are as old as the Galaxy itself.

Adopting for the PDMF $\mathrm{d} N / \mathrm{d} m \propto m^{-4.5}$ (Kroupa et al. 1993), and for the present-day star formation IMF $\mathrm{d} N / \mathrm{d} m \propto$ $m^{-2.3 \pm 0.7}$ for $1.3 M_{\odot} \leq m \leq 1.5 M_{\odot}$, and $\mathrm{d} N / \mathrm{d} m \propto m^{-2.7 \pm 0.3}$ for $0.8 M_{\odot} \leq m \leq 1 M_{\odot}$ (Kroupa 2001), we have:

$\frac{p_{1}}{p_{2}}=\frac{\int_{1.3 M_{\odot}}^{1.5 M_{\odot}} \mathrm{IMF} \mathrm{d} m}{0.8 \int_{1.3 M_{\odot}}^{1.5 M_{\odot}} \mathrm{PDMF} \mathrm{d} m} \times \frac{\int_{0.8 M_{\odot}}^{1 M_{\odot}} \mathrm{PDMF} \mathrm{d} m}{\int_{0.8 M_{\odot}}^{1 M_{\odot}} \mathrm{IMF} \mathrm{d} m}=3.18_{-0.72}^{+1.03}$

\subsubsection{With selective evaporation}

$\mathrm{N}$-body simulations of the dynamical evolution of open clusters with an initial number of 1000 stars (Terlevich 1987) have demonstrated that evaporation is more effective for low-mass stars because of mass segregation. This trend is clear in numerical simulations if one compares the relative depletion of stars lighter and heavier than $m \sim 0.5 M_{\odot}$. However, if one compares the relative number of escapers from our two mass groups after $75 \%$ of the stars have left the cluster, the effect is not clear at all (see Fig. 2 of Terlevich 1987). The numerical noise is high, and the depletion is favoured in one group or the other depending on the initial conditions. The simulations indicate that the relative number of escapers in Group 2 could be higher than in Group 1 at the very most by a factor $\kappa=1.4$. While such an efficient selective evaporation would steepen the observed mass function of stars evaporated from the cluster to bring it closer to the PDMF (and would decrease the predicted ratio $p_{1} / p_{2}$ by a factor $\kappa$ ), it would actually also flatten the observed mass function of the cluster itself. From Fig. 8 of Reid \& Hawley (1999), it appears that (for the mass range under consideration here), the Hyades cluster MF has a power-law exponent $\sim-2.3$. A factor $\kappa$ for the relative number of escapers in the range $-0.1<\log (m)<0$ as compared to the relative number of escapers in the range $0.1<\log (m)<0.2$ would then lead to an IMF with a power-law exponent of $-2.3-5 \log (\kappa)$. An extreme scenario, combining a selective evaporation with a maximal $\kappa=1.4$ and the steepest IMF $\propto m^{-3.0}$ (allowed by Kroupa 2001 error bars) is thus not formally excluded. In that extreme case, the lower bound in Eq. (1) (corresponding to an IMF power-law exponent of -3.0) should be divided by $\kappa=1.4$, thus yielding $p_{1} / p_{2}=1.76$.

\subsection{Observational test of coevality}

Let us now determine the observed value of $p_{1}$ and $p_{2}$ from the Geneva-Copenhagen survey. In order to estimate $N_{\text {hya }} / N_{\text {field }}$ in the two mass groups, we need to separate the Hyades stream from the field of the galactic disk. We therefore consider the strip $-21 \leq V\left(\mathrm{~km} \mathrm{~s}^{-1}\right) \leq-12$ in the $U V$-plane, corresponding to the range in $V$ covered by the Hyades stream (see Fig. 2). We only consider stars with $[\mathrm{Fe} / \mathrm{H}]>-0.5$ to avoid contamination by the halo (the Hyades overdensity is not seen in the $U V$-plane for stars with $[\mathrm{Fe} / \mathrm{H}]<-0.5)$.

Figures 3 and 4 display the histogram along $U$ in the $V$-strip for Groups 1 and 2, respectively. The relative Poisson noise is larger for Group 2 due to the smaller number of stars. Neglecting the Castor moving group appearing as a sharp peak in the $U$-bin 


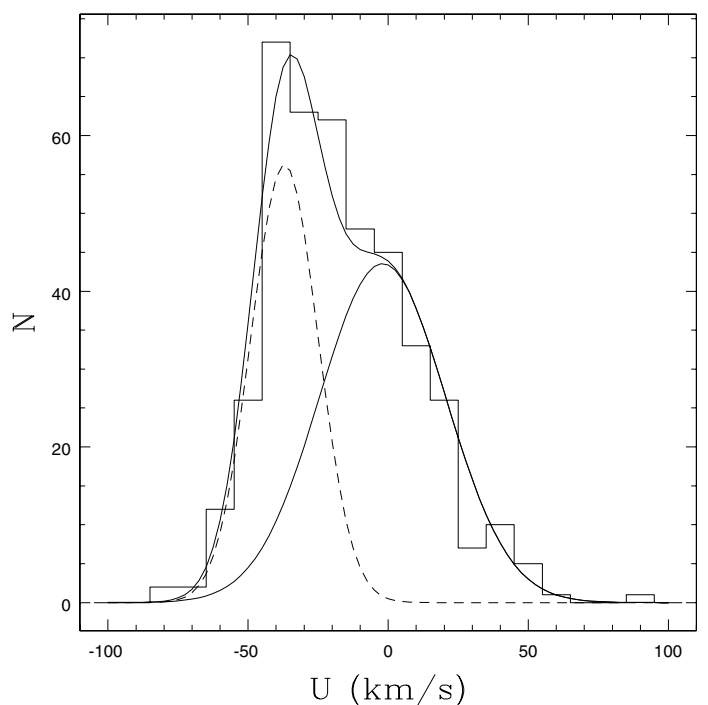

Fig. 3. Histogram of the $U$ velocities for the 415 stars in Group 1, with $-21 \leq V\left(\mathrm{~km} \mathrm{~s}^{-1}\right) \leq-12$. The dashed line corresponds to the contribution of the Hyades stream.

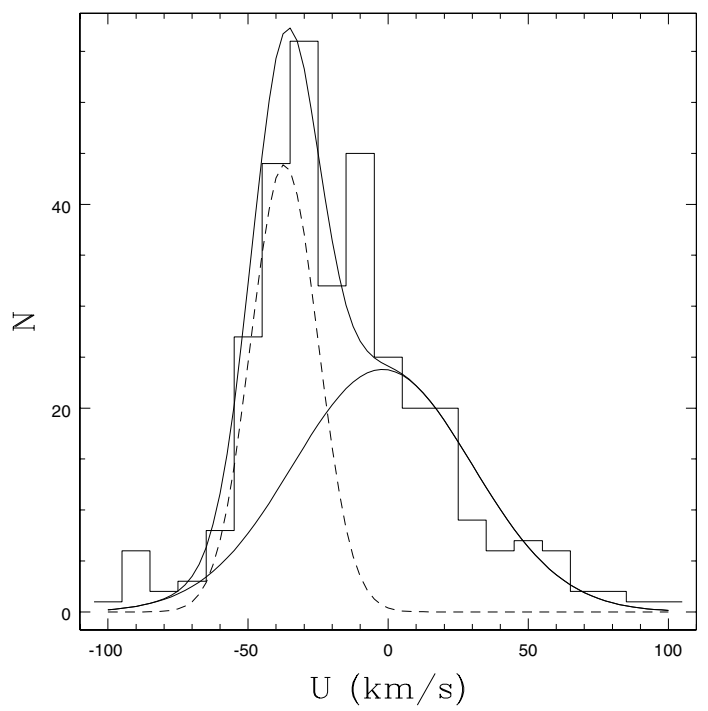

Fig. 4. Same as Fig. 3 for the 323 stars in Group 2. The sharp excess in the $U$-bin $[-15,-5]$ is due to the presence of the Castor moving group, and to a high relative Poisson noise.

$[-15,-5]$ in Group 2, these histograms may be represented by a superposition of two normalized Gaussians $\mathcal{N}\left(\langle U\rangle, \sigma_{U}\right)$, the first one corresponding to the Hyades and the second one to the field of the galactic disk:

$\tilde{N}_{1}\left(k_{1}\right)=\bar{N}_{1}\left[\left(1-k_{1}\right) \times \mathcal{N}(-37,12)+k_{1} \times \mathcal{N}(-2,22.5)\right]$

for Group 1, and

$\tilde{N}_{2}\left(k_{2}\right)=\bar{N}_{2}\left[\left(1-k_{2}\right) \times \mathcal{N}(-37,12)+k_{2} \times \mathcal{N}(-2,32)\right]$

for Group 2, where $\bar{N}_{1}, \bar{N}_{2}$ are the total number of stars of the considered group in the $V$-strip, multiplied by the width of the $U$-bins, and $k_{1}, k_{2}$ the fraction belonging to the field. The mean value $\langle U\rangle=-2 \mathrm{~km} \mathrm{~s}^{-1}$ for field stars corresponds to the value found for giant stars in Famaey et al. (2005) when the streams were not taken into account. The velocity dispersion of the field increases with decreasing mass $\left(\sigma_{U}=22.5 \mathrm{~km} \mathrm{~s}^{-1}\right.$ in Group 1 and $\sigma_{U}=32 \mathrm{~km} \mathrm{~s}^{-1}$ in Group 2), as expected from the agevelocity dispersion relation (see e.g. Dehnen \& Binney 1998).

The parameters $k_{1}$ and $k_{2}$ have been derived numerically (separately for Group 1 and 2: $j=1,2$ ) by minimizing:

$\chi^{2}\left(k_{j}\right)=\Sigma_{i=1}^{M} \frac{\left[N_{i}-\tilde{N}_{i j}\left(k_{j}\right)\right]^{2}}{\tilde{N}_{i j}\left(k_{j}\right)}$,

where the sum extends over the $M$ bins in $U, N_{i}$ is the observed number of stars in bin $i$, and the denominator $\tilde{N}_{i j}$ corresponds to the expected number of stars in bin $i$ for Group $j$. In Group 2, the bin containing Castor has not been included in the sum. Pearson's $\chi^{2}$ goodness-of-fit criterion used here should only be applied to bins containing at least 5 stars. Therefore, the fitting process has been restricted to the range $-65 \leq U\left(\mathrm{~km} \mathrm{~s}^{-1}\right) \leq 55$.

The uncertainties $\sigma_{k_{1}}, \sigma_{k_{2}}$ on the parameters $k_{1}, k_{2}$ (listed in Table 1) have been estimated from the relation

$\sigma_{k_{j}}^{2}=\Sigma_{i=1}^{M} N_{i}\left(\frac{\partial k_{j}}{\partial N_{i}}\right)^{2}$

where the derivatives $\partial k_{j} / \partial N_{i}$ are derived from the condition $\partial \chi^{2} / \partial k_{j}=0$, yielding (for Group 1)

$\sigma_{k_{1}}^{2}=\left(\Sigma_{i=1}^{M} \frac{N_{i}}{\left[k_{1}+\frac{\mathcal{N}(-37,12)}{\mathcal{N}(-2,22.5)-\mathcal{N}(-37,12)}\right]^{2}}\right)^{-1}$

under the assumption that the number of stars in the bins are large enough to ensure that $\left|N_{i}-\tilde{N}_{i j}\right| / \tilde{N}_{i j} \ll 1$.

In order to derive $p_{1}$ and $p_{2}$, we need to estimate the proportion of field stars in the considered strip $-21 \leq V\left(\mathrm{~km} \mathrm{~s}^{-1}\right) \leq-12$ with respect to the whole $U V$-plane. To avoid contamination by other streams present in our sample, we simply approximate the field-star $V$-distribution by $\mathcal{N}(-10,12)$ for Group 1 and $\mathcal{N}(-15,18)$ for Group 2 , according to the age-velocity dispersion relation and the asymmetric drift (Dehnen \& Binney 1998). We thus obtain

$p_{1}=\left(1-k_{1}\right) / k_{1} \times \int_{-21}^{-12} \mathcal{N}(-10,12) \mathrm{d} V=0.175 \pm 0.024$,

and

$p_{2}=\left(1-k_{2}\right) / k_{2} \times \int_{-21}^{-12} \mathcal{N}(-15,18) \mathrm{d} V=0.136 \pm 0.025$,

where the errors have been propagated according to $\sigma_{p}=$ $\sigma_{k}|\mathrm{~d} p / \mathrm{d} k|=\sigma_{k} / k^{2} \int_{-21}^{-12} \mathcal{N} \mathrm{d} V$. We thus have:

$\frac{p_{1}}{p_{2}}=1.29_{-0.35}^{+0.50}$.

In order to evaluate the sensitivity of these results to the choice of the $V$ strip ([-21,-12] so far), Table 1 lists $k_{1}, k_{2}, p_{1}, p_{2}$ for slightly different choices of this strip. All these choices actually yield very similar results for the $p_{1} / p_{2}$ ratio, clearly incompatible with the standard coeval scenario without selective evaporation, predicting $3.18_{-0.72}^{+1.03}$ (see Eq. (1)). Even the most favourable coeval scenario, with a steep IMF of power-law exponent -3.0 , and with a significant preferential evaporation of $1 M_{\odot}$ stars over $1.5 M_{\odot}$ stars, yields $p_{1} / p_{2}=1.76$, a ratio higher than the observed value by one sigma. In this scenario, the mass of the stream (around $800 M_{\odot}$ in the Geneva-Copenhagen catalogue alone, i.e. a lower bound since it contains only FG dwarfs) implies a very massive primordial Hyades cluster. 
Table 1. Sensitivity of the parameters characterizing the relative fraction of Hyades and field stars to the choice of the $V$ strip enclosing the Hyades (see Eqs. (2)-(4), (7) and (8) for the definition of the various parameters listed). For Group 1, Pearson's goodness-of-fit $\chi^{2}\left(k_{1}\right)$ behaves roughly like a $\chi^{2}$ distribution with 10 degrees of freedom (12 bins -2 constraints). For Group 2, that number reduces to 9 (because of the exclusion of Castor's bin). The probability that a random variable exceeds the observed $\chi^{2}$ value is in all cases large enough for the two-Gaussian model to be considered as a satisfactory fit to the data (to fix the ideas, $\operatorname{Prob}\left(\chi_{10}^{2}>17.4\right)=6.6 \%$ and $\left.\operatorname{Prob}\left(\chi_{9}^{2}>13.7\right)=13.4 \%\right)$.

\begin{tabular}{cccccccc}
\hline \hline $\begin{array}{c}V \text { strip } \\
\left(\mathrm{km} \mathrm{s}^{-1}\right)\end{array}$ & $k_{1}$ & $\chi^{2}\left(k_{1}\right)$ & $k_{2}$ & $\chi^{2}\left(k_{2}\right)$ & $p_{1}$ & $p_{2}$ & $p_{1} / p_{2}$ \\
\hline$[-21,-12]$ & $0.592 \pm 0.033$ & 13.8 & $0.591 \pm 0.045$ & 11.3 & $0.175 \pm 0.024$ & $0.136 \pm 0.025$ & $1.29_{-0.35}^{+0.50}$ \\
{$[-20,-13]$} & $0.572 \pm 0.037$ & 17.4 & $0.566 \pm 0.051$ & 11.0 & $0.190 \pm 0.029$ & $0.151 \pm 0.031$ & $1.26_{-0.37}^{+0.57}$ \\
{$[-22,-13]$} & $0.588 \pm 0.033$ & 16.6 & $0.606 \pm 0.046$ & 9.9 & $0.178 \pm 0.024$ & $0.128 \pm 0.025$ & $1.39_{-0.38}^{+0.57}$ \\
{$[-20,-11]$} & $0.595 \pm 0.034$ & 14.3 & $0.576 \pm 0.046$ & 13.7 & $0.173 \pm 0.024$ & $0.145 \pm 0.027$ & $1.19_{-0.33}^{+0.48}$ \\
\hline
\end{tabular}

Chumak et al. (2005) showed that, after $600 \mathrm{Myr}, 800 M_{\odot}$ could be lost by the cluster if the initial mass was $1400 M_{\odot}$ and the initial virial radius $7.5 \mathrm{pc}$ : this evaporated mass would be distributed in a volume of radius $300 \mathrm{pc}$ around the Sun, but since $800 M_{\odot}$ is observationally a lower bound, even more extreme initial conditions would probably be needed to account for the full Hyades stream. We thus conclude that the most likely scenario is that the Hyades overdensity in the $U V$-plane is not uniquely composed of coeval stars evaporated from the primordial Hyades cluster.

Note that the zeroth order expectation $p_{1}=p_{2}$ for a stream exclusively composed of field stars is well within the error bars of Eq. (9). However, the best fit value yields $p_{1}>p_{2}$. There might be several reasons for this:

- since the ratio of Eq. (9) was observationally estimated under the coevality hypothesis, we did not take into account possible variations of the intrinsic velocity dispersion of the Hyades stream itself. However, under the dynamical perturbation hypothesis, the Hyades stream itself could be affected by the age-velocity dispersion relation. For instance, if we take for the Hyades $\sigma_{U}=15 \mathrm{~km} \mathrm{~s}^{-1}$ instead of $12 \mathrm{~km} \mathrm{~s}^{-1}$ in the older Group 2 (Eq. (3)), we find $p_{1} / p_{2}=1.15_{-0.32}^{+0.47}$, a value closer to one than Eq. (9).

- $p_{1}>p_{2}$ might be caused by the PDMF of the Hyades stream being slightly different from the PDMF of the solar neighbourhood. As we shall see in the next section, the Hyades stream is more metal-rich than the solar neighbourhood at all masses, pointing towards an origin in the inner Galaxy. Since the lifetime of stars depends on the metallicity, the PDMF of the inner Galaxy should be slightly different from the PDMF of the solar neighbourhood, even with identical IMF and stellar formation rates (SFR). Moreover, the SFR could also vary as a function of the galactocentric radius.

- $p_{1}>p_{2}$ might be due to some of the stars in the stream still belonging to the evaporated cluster since we know that the Hyades cluster is kinematically associated with the Hyades stream, and we also know that this cluster does evaporate with time and must create a velocity clump of spatially extended stars. Assuming the same PDMF for the Hyades stream and the solar neighbourhood (zeroth order approximation), and the best fit value for the ratio $p_{1} / p_{2}$, we can estimate what proportion of stars in each group is associated with the coeval Hyades cluster, and what proportion is associated with the dynamical stream. Eq. (1) gives the ratio of the evaporated cluster proportion in Group 1 with respect to this proportion in Group 2, while the ratio is assumed to be 1 for the dynamical stream. Equations (7) and (8) then give the sum of these proportions in each group. From there we find that the evaporated cluster represents about $40 \%$ of the total stream in Group 1, but only $15 \%$ in Group 2. These numbers are given here as an indication since these proportions should be affected by the variation of the stream PDMF, and could be zero within the error bars of Eq. (9).

\subsection{Metallicity of the stream}

We have thus shown that the vast majority of stars in the Hyades stream are most likely field-like disk stars obeying the PDMF of the disk. This does not necessarily imply that the stream originates from a non-axisymmetric perturbation of the Galactic potential. One could instead imagine that the primordial Hyades open cluster was an extremely massive object able to trap some older galactic field stars during its formation process (see e.g. Fellhauer et al. 2006). These stars could have formed a dynamically hotter sub-system in the outer part of the object, and could have been preferentially evaporated (whilst obeying the PDMF). However, in that case, one would expect the stream to exhibit a slightly sub-solar metallicity, characteristic of the solar galactocentric radius, close to which the Hyades cluster is supposed to have formed if it has not been dynamically perturbed by spiral arms.

The mean metallicity of the Geneva-Copenhagen survey is $[\mathrm{Fe} / \mathrm{H}]=-0.16$. Excluding halo stars with $[\mathrm{Fe} / \mathrm{H}]<-0.5$, and excluding all the stars from the Hyades box $\left(-21 \mathrm{~km} \mathrm{~s}^{-1} \leq V \leq\right.$ $-12 \mathrm{~km} \mathrm{~s}^{-1}$ and $-50 \mathrm{~km} \mathrm{~s}^{-1} \leq U \leq-25 \mathrm{~km} \mathrm{~s}^{-1}$ ), we get a mean disk metallicity of $[\mathrm{Fe} / \mathrm{H}]=-0.126 \pm 0.002$ for the solar neighbourhood (the error representing the standard error of the mean).

If we rather concentrate on the Hyades box, we get $[\mathrm{Fe} / \mathrm{H}]=$ $-0.061 \pm 0.012$ for Group 1 , and $[\mathrm{Fe} / \mathrm{H}]=-0.059 \pm 0.013$ for Group 2, meaning that the stream is over metal-rich at all masses. We can calculate the ratio of stars from the stream to stars from the field disk in the Hyades box from Figs. 3 and 4. We get $N_{\text {hya }} / N_{\text {field }}($ box) $=3.65$ for Group 1, and 2.88 for Group 2, meaning that the stream has a roughly constant mean metallicity $[\mathrm{Fe} / \mathrm{H}]=+0.02$, even slightly rising for low-mass stars. This is inconsistent with the hypothesis of a field population from the solar neighbourhood trapped in the primordial cluster and subsequently evaporated.

On the other hand, this constancy of the metallicity is consistent with the assumption that evaporated and field-like stars in the stream have roughly the same metallicity. This could imply that they both come from the inner regions of the Galaxy after having crossed the corotation of the spiral pattern (the cluster could have been shifted in radius while remaining bound since the effect of a spiral wave on stars depends on the stars' phase with respect to the spiral, and the phase does not vary much across the cluster). The metallicity excess of 0.15 dex is indeed not consistent with the present-day orbit of the Hyades 
stream: this present-day orbit is centered on a guiding radius $R_{\mathrm{g}} \sim 7.5 \mathrm{kpc}$ in galactocentric coordinates $(8 \mathrm{kpc}$ being the galactocentric radius of the Sun), and would imply an implausible metallicity gradient of $-0.3 \mathrm{dex} / \mathrm{kpc}$ in the disk. Assuming a very steep but realistic (see e.g. Daflon \& Cunha 2004) galactic metallicity gradient of $-0.07 \mathrm{dex} / \mathrm{kpc}$, the metallicity excess is compatible with a galactocentric origin near $R=6 \mathrm{kpc}$. This would be consistent with the order of magnitude of stellar wandering caused by spiral perturbations ( $2-3 \mathrm{kpc}$, see Sellwood \& Binney 2002; Lépine et al. 2003). A flatter metallicity gradient would imply an even more internal origin for the stream.

\section{Discussion}

Using the Geneva-Copenhagen catalogue of $F$ and $G$ dwarfs (Nordström et al. 2004), we have analyzed the mass function of the Hyades stream, an overdensity of stars in the $U V$-plane kinematically associated with the Hyades cluster.

In order to be compatible at a one-sigma level with the predicted mass function for stars evaporated from the primordial Hyades cluster, one needs a rather extreme scenario, with a very significant preferential evaporation from the cluster of $1 M_{\odot}$ stars over $1.5 M_{\odot}$ stars, and with a very steep IMF, not flatter than a power-law exponent of -3.0 (see Sects. 4.1 and 4.2). The initial total mass and virial radius of the cluster should also be very high $\left(M>1400 M_{\odot}, r>7.5 \mathrm{pc}\right)$.

On the other hand, the observed mass function (see Sect. 4.2, Eq. (9)) is also compatible at a one-sigma level with the hypothesis that the stream is uniquely composed of field-like stars obeying the PDMF. However, because the Hyades cluster is known to share the kinematics of the stream and to evaporate over time, the most likely scenario is that the stream is indeed mainly composed of field-like stars (about $85 \%$ of the stream for low-mass stars), but also partly of coeval stars evaporated from the primordial Hyades cluster (about 15\% of the stream for low-mass stars).

The peculiar metallicity of the stream at all masses (see Sect. 4.3) is not compatible with a scenario where the field-like stars would have been trapped in the primordial Hyades cluster during its formation process, and subsequently evaporated. Those stars were thus most probably trapped at resonance by a spiral perturbation. Indeed, a series of strong transient spirals with their mean corotation at the solar galactocentric radius are known to produce small-scale structure in the local velocity distribution (De Simone et al. 2004). Stars on horseshoe orbits that cross the corotation could wander over $2-3 \mathrm{kpc}$ in much less than 1 Gyr (Sellwood \& Binney 2002; Lépine et al. 2003), while staying on quasi-circular orbits, not elongated enough to betray their place of birth. This is consistent with a galactocentric origin near $R=6 \mathrm{kpc}$ for the Hyades stream, that could explain its metallicity excess at low masses. On the other hand, the Hyades stream could also correspond to nearly closed orbits trapped at the $4: 1$ inner Lindblad resonance of a two-armed spiral density wave (Quillen \& Minchev 2005). In any case, the prominence of the Hyades dynamical stream raises the question of the amplitude of the spiral perturbation needed to produce such a stream. The most detailed models of gas flows in the Galaxy (Bissantz et al. 2003) indicate that the amplitude of the spiral structure in the mass density is larger by a factor of 1.5 than its amplitude in the near-infrared luminosity density, while such a large amplitude is also needed to produce the large non-axisymmetric motion of the star-forming region $\mathrm{W} 3 \mathrm{OH}$ (Xu et al. 2006). This requires the baryonic disk to be massive even near the Sun, and is very constraining for the dark matter distribution in the Galaxy (see e.g. Famaey \& Binney 2005). On the other hand, the probable resonant origin of the Hyades stream reveals the importance of evaluating the impact of radial migrations on the chemical evolution of the Galaxy (see also Haywood 2006).

Acknowledgements. We thank the referee Michel Crézé for many helpful suggestions that have considerably improved the paper. B.F. is an FNRS Research Associate, A.J. is an FNRS Senior Research Associate. X.L. acknowledges the support from the Spanish MCyT through grant AYA2003-07736.

\section{References}

Baumgardt, H., \& Makino, J. 2003, MNRAS, 340, 227

Bissantz, N., Englmaier, P., \& Gerhard, O. 2003, MNRAS, 340, 949

Chereul, E., Crézé, M., \& Bienaymé, O. 1999, A\&A, S135, 5

Chereul, E., \& Grenon, M. 2001, in Dynamics of star clusters and the Milky Way, ed. S. Deiters, B. Fuchs, A. Just, R. Spurzem, \& R. Wielen, ASP Conf., 228, 398

Chumak, Ya. O., Rastorguev, A. S., \& Aarseth, S. J. 2005, AstL, 31, 308

Daflon, S., \& Cunha, K. 2004, ApJ, 617, 1115

de Bruijne, J., Hoogerwerf, R., \& de Zeeuw, P. T. 2001, A\&A, 367, 111

Dehnen, W. 1998, AJ, 115, 2384

Dehnen, W. 2000, AJ, 119, 800

Dehnen, W., \& Binney, J. J. 1998, MNRAS, 298, 387

De Simone, R. S., Wu, X., \& Tremaine, S. 2004, MNRAS, 350, 627

Dobbie, P. D., Kenyon, F., Jameson, R. F., et al. 2002, MNRAS, 329, 543

Eggen, O. J. 1958, MNRAS, 118, 65

Famaey, B., \& Binney, J. J. 2005, MNRAS, 363, 603

Famaey, B., Jorissen, A., Luri, X., et al. 2005, A\&A, 430, 165

Fellhauer, M., Kroupa, P., \& Evans, W. 2006, MNRAS, 372, 338

Fux, R. 2001, A\&A, 373, 511

Girardi, L., Bressan, A., Bertelli, G., \& Chiosi, C. 2000, A\&AS, 141, 371

Haywood, M. 2006, MNRAS, 371, 1760

Helmi, A., Navarro, J., Nordström, B., et al. 2006, MNRAS, 365, 1309

Hertzsprung, E. 1909, ApJ, 30, 135

Kroupa, P. 2001, MNRAS, 322, 231

Kroupa, P., Tout, C. A., \& Gilmore, G. 1993, MNRAS, 262, 545

Lejeune, T., \& Schaerer, D. 2001, A\&A, 366, 538

Lépine, J. R. D., Acharova, I. A., \& Mishurov, Y. N. 2003, ApJ, 589, 210

Montes, D., Lopez-Santiago, J., Galvez, M. C., et al. 2001, MNRAS, 328, 45

Nordström, B., Mayor, M., Andersen, J., et al. 2004, A\&A, 418, 989

Perryman, M. A. C, Brown, A. G. A, Lebreton, Y., et al. 1998, A\&A, 331, 81

Pont, F., \& Eyer, L. 2004, MNRAS, 351, 487

Quillen, A. C., \& Minchev, I. 2005, AJ, 130, 576

Reid, I. N., \& Hawley, S. L. 1999, AJ, 117, 343

Sellwood, J. A., \& Binney, J. J. 2002, MNRAS, 336, 785

Strömberg, G. 1922, ApJ, 56, 265

Terlevich, E. 1987, MNRAS, 224, 193

Woolley, R. 1961, The Observatory, 81, 203

Xu, Y., Reid, M. J., Zheng, X. W., \& Menten, K. M. 2006, Science, 311, 54 